\title{
Sampled-Data Control of Singular Systems with Time Delays
}

\author{
Zheng Minjie, ${ }^{1,2}$ Zhou Yujie, $^{1}$ Yang Shenhua, ${ }^{2}$ and Li Lina ${ }^{2}$ \\ ${ }^{1}$ Shanghai Jiao Tong University, Shanghai, China \\ ${ }^{2}$ Jimei University, No. 1 Jiageng Road, Jimei, Xiamen, 361000 Fujian, China \\ Correspondence should be addressed to Yang Shenhua; yangshh@163.com
}

Received 24 November 2013; Revised 26 January 2014; Accepted 13 February 2014; Published 3 April 2014

Academic Editor: Qun Lin

Copyright (C) 2014 Zheng Minjie et al. This is an open access article distributed under the Creative Commons Attribution License, which permits unrestricted use, distribution, and reproduction in any medium, provided the original work is properly cited.

\begin{abstract}
This paper is concerned with sampled-data controller design for singular systems with time delay. It is assumed that the sampling periods are arbitrarily varying but bounded. A time-dependent Lyapunov function is proposed, which is positive definite at sampling times but not necessarily positive definite inside the sampling intervals. Combining input delay approach with Lyapunov method, sufficient conditions are derived which guarante that the singular system is regular, impulse free, and exponentially stable. Then, the existence conditions of desired sampled-data controller can be obtained, which are formulated in terms of strict linear matrix inequality. Finally, numerical examples are given to demonstrate the effectiveness and the benefits of the proposed method.
\end{abstract}

\section{Introduction}

In the last decade, considerable attention has been devoted to sampled-data control systems, because modern control systems usually employ digital technology for controller implementation [1-8]. The systems can adopt a digital computer to sample and quantize a continuous-time measurement signal to produce a discrete-time control input signal, which will be converted back into a continuous-time control input signal using a zero-order hold $(\mathrm{ZOH})$ [9]. Recently, three main approaches have been adopted to analyze the sampled-data systems. The first one is based on discrete-time models [9]. The second one is based on the representation of the sampleddata system in the form of impulsive model. The impulsive model approach was applied to sampled-data stabilization of linear uncertain systems in the case of constant sampling, where a piecewise linear in time Lyapunov function was suggested [10]. The third one is the input delay approach [11], where the system is modeled as a continuous-time system with the delayed control input, and it is popular and has been widely adopted in sampled-data systems [11-15]. In [16], a novel time dependent Lyapunov functional-based technique for sampled-data control has been introduced in the framework of the input delay approach. The most significant advantage of the method is that the sawtooth evolution of the time-varying delay induced by sample and hold is used. Thus, Recently, the time-dependent Lyapunov functional method has been applied to all sorts of sampleddata systems, and some useful results have been obtained (see, e.g., $[17-24]$ and the references therein).

On the other hand, singular systems, also referred to as descriptor systems, generalized state-space systems, differential-algebraic systems, or semistate systems, provide convenient and natural representations in the description of economic systems, power systems, and circuits systems [2530 ], and it have been extensively studied in the past few years due to the fact that singular systems better describe physical systems than state-space ones. Apparently, in nowadays digitalized world, it is of both theoretical significance and practical importance to analyze how a digitalized control signal would influence the singular systems. In other words, there is a vital need to investigate the sampled-data control for singular systems. Unfortunately, although sampled-data control technologies have been developed relatively well in control theory, the particular sampled-data control for singular systems has so far received very little attention mainly due to the mathematical complexity. Indeed, the essential difficulties would be (1) how to deal with the obtained results to guarantee the considered singular systems not only to be stable but also to be regular and impulse free in order to ensure the existence, uniqueness, and absence of impulses of a solution to a given system, (2) how to fully adopt the available 
information about the actual sampling pattern, and (3) how to actually design a set of easy-to-implement sampled-data controllers in order to guarantee that the singular systems are exponentially stable. It is, therefore, the main aim of this paper to challenge the sampled-data control for singular systems by overcoming the aforementioned three major difficulties.

This paper is concerned with the sampled-data control of singular systems with time delays which are important sources of oscillation, divergence, and instability in systems, and thus time-delay systems have been widely studied recently [31,32]. In terms of LMI approach, stability conditions are proposed to guarantee the considered system to be regular, impulse free, and exponentially stable. In order to make full use of the available information about the actual sampling pattern, a time-dependent Lyapunov functional is proposed. The positive definitiveness of the given Lyapunov functional is required only at sampling times but not necessarily inside the sampling intervals. Two numerical examples are given to illustrate the effectiveness of the methods given in the paper.

Notation. Throughout this paper, the superscripts " $T$ " and " -1 " stand for the transpose of a matrix and the inverse of a matrix. $R^{n}$ denotes the $n$-dimensional Euclidean space, and $R^{n \times m}$ is the set of $n \times m$ real matrices. The notation $X>Y(X \geq$ $Y$ ), where $X$ and $Y$ are symmetric matrices, means that $X-Y$ is positive definite (positive semidefinite). $I$ is the identity matrix of appropriate dimensions. $\|\cdot\|$ denotes the Euclidean norm of a vector and its induced norm of a matrix. $\lambda_{\max }(P)$ and $\lambda_{\min }(P)$ refer to the maximal and minimal eigenvalues of the matrix $P$, respectively. For a symmetric matrix, “*” denotes the matrix entries implied by symmetry.

\section{Problem Formulation}

Consider the following sampled-data control of singular system:

$$
\begin{gathered}
E \dot{x}(t)=A x(t)+A_{d} x(t-d)+u(t), \quad t>0 \\
x(t)=\phi(t), \quad t \in[-d, 0],
\end{gathered}
$$

where $x(t) \in R^{n}$ is the state vector, $u(t) \in R^{n}$ is the control input, and the initial condition, $\phi(t)$, is a continuous vector valued initial function of $t \in[-d, 0] . E, A$, and $A_{d}$ are known matrices of appropriate dimensions, where $E \in R^{n \times n}$ may be singular, and we assume that $\operatorname{rank} E=r \leq n$. $d$ is a given time delay.

In this paper, it is assumed that we only have the measurement $x\left(t_{k}\right)$ at the sampling instant $t_{k}$; that is, only discrete measurements of $x(t)$ are available for control purposes, and the control signal is assumed to be generated by using a zeroorder-hold $(\mathrm{ZOH})$ function with a sequence of hold times:

$$
0=t_{0}<t_{1}<\cdots<t_{k}<\cdots<\lim _{k \rightarrow \infty} t_{k}=+\infty .
$$

Also, the sampling is not required to be periodic, and the only assumption is that the distance between any two consecutive sampling instants is less than a given bound. It is assumed that

$$
t_{k+1}-t_{k}=h_{k} \leq h
$$

for all $k \geq 0$, where $h>0$ represents the upper bound of the sampling periods. Then, for system (1), we consider a statefeedback control law of the form

$$
u(t)=K x\left(t_{k}\right), \quad t_{k} \leq t<t_{k+1},
$$

where $K$ is the local gain matrix of the state feedback controller to be determined.

By substituting (4) into (1), we obtain

$$
\begin{gathered}
E \dot{x}(t)=A x(t)+A_{d} x(t-d)+K x\left(t_{k}\right), \quad t>0 \\
x(t)=\phi(t), \quad t \in[-d, 0] .
\end{gathered}
$$

Throughout this paper, we will use the following concepts.

\section{Definition 1.}

(1) The pair $(E, A)$ is said to be regular if $\operatorname{det}(s E-A)$ is not identically zero.

(2) The pair $(E, A)$ is said to be impulse free if $\operatorname{deg}(\operatorname{det}(s E-A))=\operatorname{rank} E$.

Definition 2 (see [33]).

(1) The sampled-data control of singular system (5) is said to be regular and impulse free if the pair $(E, A)$ is regular and impulse free.

(2) The sampled-data control of singular system (5) is said to be exponentially stable, if there exist scalars $\alpha>0$ and $\beta>0$ such that

$$
\|E x(t)\| \leq \beta e^{-\alpha t}\left\|x_{0}\right\|_{c^{\prime}}, \quad \forall t \geq 0,
$$

where $\left\|x_{0}\right\|_{\mathcal{c}}=\sup _{-d \leq \theta \leq 0}\{\|x(\theta)\|,\|E \dot{x}(\theta)\|\}$.

(3) The sampled-data control of singular system (5) is said to be exponentially admissible, if it is regular, impulse free, and exponentially stable.

Lemma 3. Given singular system (5), the following inequality holds:

$$
\|E x(t)\|^{2} \leq \theta_{1}\left\|x\left(t_{k}\right)\right\|^{2}+\theta_{2} \int_{t_{k}-d}^{t_{k}}\|x(\alpha)\|^{2} d \alpha, \quad t_{k} \leq t<t_{k+1},
$$

where $\theta_{1}=4\left(\|E\|^{2}+\|K\|^{2} h\right) e^{4\left(\|A\|^{2}+\left\|A_{d}\right\|^{2}\right)}, \theta_{2}=$ $4\left\|A_{d}\right\|^{2} e^{4\left(\|A\|^{2}+\left\|A_{d}\right\|^{2}\right)}$.

Proof. For any $t \in\left[t_{k}, t_{k+1}\right)$, it follows from (5) that

$$
\begin{aligned}
\|E x(t)\| \leq & \left\|E x\left(t_{k}\right)\right\| \\
& +\left\|\int_{t_{k}}^{t} A x(\alpha) d \alpha\right\|+\left\|\int_{t_{k}}^{t} A_{d} x(\alpha-d) d \alpha\right\| \\
& +\left\|\int_{t_{k}}^{t} K x\left(t_{k}\right) d \alpha\right\| .
\end{aligned}
$$


Applying the Cauchy-Schwarz inequality, we find from (8) that

$$
\begin{aligned}
\|E x(t)\|^{2} \leq & 4\left\|E x\left(t_{k}\right)\right\|^{2} \\
& +4\left\|\int_{t_{k}}^{t} A x(\alpha) d \alpha\right\|^{2}+4\left\|\int_{t_{k}}^{t} A_{d} x(\alpha-d) d \alpha\right\|^{2} \\
& +4\left\|\int_{t_{k}}^{t} K x\left(t_{k}\right) d \alpha\right\|^{2} .
\end{aligned}
$$

Using the Cauchy-Schwarz inequality again, we obtain from (9) that

$$
\begin{aligned}
\|E x(t)\|^{2} \leq & 4\left\|E x\left(t_{k}\right)\right\|^{2} \\
& +4 \int_{t_{k}}^{t}\|A x(\alpha)\|^{2} d \alpha+4 \int_{t_{k}}^{t}\left\|A_{d} x(\alpha-d)\right\|^{2} d \alpha \\
& +4 \int_{t_{k}}^{t}\left\|K x\left(t_{k}\right)\right\|^{2} d \alpha \\
\leq & 4\|E\|^{2}\left\|x\left(t_{k}\right)\right\|^{2}+4\|A\|^{2} \int_{t_{k}}^{t}\|x(\alpha)\|^{2} d \alpha \\
& +4\|K\|^{2} \int_{t_{k}}^{t}\left\|x\left(t_{k}\right)\right\|^{2} d \alpha \\
& +4\left\|A_{d}\right\|^{2} \int_{t_{k}}^{t}\|x(\alpha-d)\|^{2} d \alpha
\end{aligned}
$$

$$
\begin{aligned}
\leq & 4\left(\|E\|^{2}+\|K\|^{2} h\right)\left\|x\left(t_{k}\right)\right\|^{2} \\
& +4\left\|A_{d}\right\|^{2} \int_{t_{k}-d}^{t_{k}}\|x(\alpha)\|^{2} d \alpha \\
& +4\left(\|A\|^{2}+\left\|A_{d}\right\|^{2}\right) \int_{t_{k}}^{t}\|x(\alpha)\|^{2} d \alpha .
\end{aligned}
$$

Applying the Gronwall-Bellman Lemma to (10), we can obtain (7) immediately. This completes the proof.

\section{Main Results}

In this section, the exponential stability of sampled-data control for singular system (5) is first investigated based on the time-dependent Lyapunov functional approach, and sufficient condition is derived to guarantee the system stability and synthesize the sampled-data controllers in the form of (4).

Theorem 4. Given scale $\alpha>0$, the sampled-data control for singular system (5) is exponentially stable if there exist symmetric positive-definite matrices $P, F, Q, Z, U,\left[\begin{array}{cc}R_{1} & R_{2} \\ * & R_{3}\end{array}\right]>0$, $X_{j}, j=1,2,3,4,5, F_{1}, L, H=\left[\begin{array}{llll}H_{1} & H_{2} & H_{3} & H_{4}\end{array}\right]$, such that

$$
\begin{aligned}
& E^{T} F=F^{T} E \geq 0, \\
& \Xi_{1}(\bar{h})=\left[\begin{array}{ccccc}
\Xi_{11}+\Theta_{11}(\bar{h}) & \Xi_{12}+\Theta_{12}(\bar{h}) & \Xi_{13}+\Theta_{13}(\bar{h}) & \Xi_{14}+\Theta_{14}(\bar{h}) & \Xi_{15} \\
* & \Xi_{22}+\bar{h} U & \Xi_{23}+\Theta_{23}(\bar{h}) & \bar{h} E^{T} X_{3} E & F_{1} A_{d} \\
* & * & \Xi_{33}+\Theta_{33}(\bar{h}) & \Xi_{34}+2 \alpha \bar{h} X_{4} & 0 \\
* & * & * & \Xi_{44}+\Theta_{44}(\bar{h}) & 0 \\
* & * & * & * & \Xi_{55}
\end{array}\right]<0, \bar{h} \in[0, h], \\
& \Xi_{2}(\bar{h})=\left[\begin{array}{cccccc}
\Xi_{11} & \Xi_{12} & \Xi_{13} & \Xi_{14} & \Xi_{15} & \sqrt{\bar{h}} E^{T} H_{1}^{T} E \\
* & \Xi_{22} & \Xi_{23} & 0 & F_{1} A_{d} & \sqrt{\bar{h}} E^{T} H_{2}^{T} E \\
* & * & \Xi_{33}-e^{-2 \alpha h} \bar{h} R_{3} & \Xi_{34} & 0 & \sqrt{\bar{h}} E^{T} H_{3}^{T} E \\
* & * & * & \Xi_{44} & 0 & \sqrt{\bar{h}} E^{T} H_{4}^{T} E \\
* & * & * & * & \Xi_{55} & 0 \\
* & * & * & * & * & -e^{-2 \alpha h} E^{T} U E
\end{array}\right]<0 \text {, }
\end{aligned}
$$


where

$$
\begin{aligned}
& \Xi_{11}=2 \alpha E^{T} P E+Q-E^{T} \frac{X_{1}+X_{1}^{T}}{2} E-\frac{e^{-2 \alpha d}}{d} E^{T} Z E \\
& +E^{T}\left(H_{1}+H_{1}^{T}\right) E+F^{T} A+A^{T} F \\
& \Xi_{12}=E^{T} P+E^{T} H_{2} E-F^{T}+A^{T} F_{1}^{T}, \\
& \Xi_{13}=E^{T}\left(X_{1}-X_{2}\right) E+E^{T}\left(H_{3}-H_{1}^{T}\right) E+F^{T} K \text {, } \\
& \Xi_{14}=-E^{T} X_{3} E+E^{T} H_{4} E, \\
& \Xi_{15}=\frac{e^{-2 \alpha d}}{d} E^{T} Z E+F^{T} A_{d} \\
& \Xi_{22}=d Z-F_{1}-F_{1}^{T} \text {, } \\
& \Xi_{23}=-E^{T} H_{2}^{T} E-F_{1}^{T} K \text {, } \\
& \Xi_{33}=E^{T}\left(X_{2}+X_{2}^{T}-\frac{X_{1}+X_{1}^{T}}{2}\right) E-E^{T}\left(H_{3}+H_{3}^{T}\right) E \text {, } \\
& \Xi_{34}=-E^{T} X_{4} E-e^{-2 \alpha h} R_{2}^{T}-E^{T} H_{4} E^{T}, \\
& \Xi_{44}=-E^{T} \frac{X_{5}+X_{5}^{T}}{2} E-\frac{e^{-2 \alpha h}}{h} R_{1} \text {, } \\
& \Xi_{55}=-e^{-2 \alpha d} Q-\frac{e^{-2 \alpha d}}{d} E^{T} Z E, \\
& \Theta_{11}(\bar{h})=a \bar{h} E^{T}\left(X_{1}+X_{1}^{T}\right) E+\bar{h} E^{T}\left(X_{3}+X_{3}^{T}\right) E+\bar{h} R_{1}, \\
& \Theta_{12}(\bar{h})=\bar{h} E^{T} \frac{X_{1}+X_{1}^{T}}{2} E, \\
& \Theta_{13}(\bar{h})=2 \alpha \bar{h} E^{T}\left(-X_{1}+X_{2}\right) E+\bar{h} E^{T} X_{4}^{T} E+\bar{h} R_{2}, \\
& \Theta_{14}(\bar{h})=2 \alpha \bar{h} E^{T} X_{3} E+\bar{h} E^{T} \frac{X_{5}+X_{5}^{T}}{2} E, \\
& \Theta_{23}(\bar{h})=\bar{h} E^{T}\left(-X_{1}+X_{2}\right) E, \\
& \Theta_{33}(\bar{h})=2 \alpha \bar{h} E^{T}\left(-X_{2}-X_{2}^{T}+\frac{X_{1}+X_{1}^{T}}{2}\right) E+\bar{h} R_{3}, \\
& \Theta_{44}(\bar{h})=\alpha \bar{h} E^{T}\left(X_{5}+X_{5}^{T}\right) E .
\end{aligned}
$$

Proof. Since rank $E=r \leq n$, there exist nonsingular matrices $\widehat{G}$ and $\widehat{H}$ such that

$$
\bar{E}=\widehat{G} E \widehat{H}=\left[\begin{array}{cc}
I_{r} & 0 \\
0 & 0
\end{array}\right]
$$

Similar to (15), we define

$$
\bar{A}=\widehat{G} A \widehat{H}=\left[\begin{array}{ll}
A_{11} & A_{12} \\
A_{21} & A_{22}
\end{array}\right], \quad \bar{F}=\widehat{G}^{-T} F \widehat{H}=\left[\begin{array}{ll}
F_{11} & F_{12} \\
F_{21} & F_{22}
\end{array}\right] \text {. }
$$

From (11), we have $\bar{F}=\left[\begin{array}{ll}F_{11} & 0 \\ F_{21} & F_{22}\end{array}\right]$, and $F_{11}=F_{11}^{T}>0$.
Premultiplying and postmultiplying $\Xi_{11}<0$ by $\widehat{H}^{T}$ and $\widehat{H}$, respectively, we have $A_{22}^{T} F_{22}+F_{22}^{T} A_{22}<0$, which implies that $A_{22}$ is nonsingular and the pair $(E, A)$ is regular and impulse free. Then, by Definition 2 , the sampled-data control for singular system (5) is regular and impulse free.

Next, we will show the exponential stability of system (5). Consider the following Lyapunov functional of sampled-data control for singular system (5):

$$
\begin{aligned}
& V(t)=\sum_{i=1}^{6} V_{i}(t), \quad t \in\left[t_{k}, t_{k+1}\right), \\
& V_{1}(t)=e^{2 \alpha t} x(t)^{T} E^{T} \operatorname{PEx}(t), \\
& V_{2}(t)=\int_{t-d}^{t} e^{2 \alpha s} x(s)^{T} Q x(s) d s, \\
& V_{3}(t)=\int_{-d}^{0} \int_{t+\theta}^{t} e^{2 \alpha s} \dot{x}(s)^{T} E^{T} Z E \dot{x}(s) d s d \theta, \\
& V_{4}(t)=\left(t_{k+1}-t\right) \int_{t_{k}}^{t} e^{2 \alpha s} \dot{x}(s)^{T} E^{T} U E \dot{x}(s) d s, \\
& V_{5}(t)=\left(t_{k+1}-t\right) \int_{t_{k}}^{t} e^{2 \alpha s}\left[\begin{array}{c}
x(s) \\
x\left(t_{k}\right)
\end{array}\right]^{T}\left[\begin{array}{cc}
R_{1} & R_{2} \\
* & R_{3}
\end{array}\right]\left[\begin{array}{c}
x(s) \\
x\left(t_{k}\right)
\end{array}\right] d s, \\
& V_{6}(t)=\left(t_{k+1}-t\right) e^{2 \alpha t}\left[\begin{array}{c}
x(s) \\
\int_{t_{k}}^{t} x(s) d s \\
x\left(t_{k}\right)
\end{array}\right]^{T} E^{T} \Gamma E\left[\begin{array}{c}
x(s) \\
\int_{t_{k}}^{t} x(s) d s \\
x\left(t_{k}\right)
\end{array}\right] \text {, } \\
& \Gamma=\left[\begin{array}{ccc}
\frac{X_{1}+X_{1}^{T}}{2} & X_{3} & -X_{1}+X_{2} \\
* & \frac{X_{5}+X_{5}^{T}}{2} & X_{4} \\
* & * & -X_{2}-X_{2}^{T}+\frac{X_{1}+X_{1}^{T}}{2}
\end{array}\right]
\end{aligned}
$$

It is noted that, similar to [16], we have

$$
\lim _{t \rightarrow t_{k}^{+}} V_{j}(t)=\lim _{t \rightarrow t_{k}^{-}} V_{j}(t)=V_{j}\left(t_{k}\right)=0, \quad j=4,5,6
$$

Therefore, $V(t)$ is continuous in time since $\lim _{t \rightarrow t_{k}} V(t)=$ $V\left(t_{k}\right)$. Calculating the time derivative of $V(t)$ along the trajectories of (5) gives the following result:

$$
\begin{aligned}
\dot{V}_{1}(t)= & 2 e^{2 \alpha t} x(t)^{T} E^{T} P E \dot{x}(t) \\
& +2 \alpha e^{2 \alpha t} x(t)^{T} E^{T} P E x(t), \\
\dot{V}_{2}(t)= & e^{2 \alpha t} x(t)^{T} Q x(t)-e^{2 \alpha t} e^{-2 \alpha d} \\
& \times x(t-d)^{T} Q x(t-d),
\end{aligned}
$$




$$
\begin{aligned}
& \dot{V}_{3}(t)=d e^{2 \alpha t} \dot{x}(t)^{T} E^{T} Z E \dot{x}(t) \\
& -\int_{t-d}^{t} e^{2 \alpha s} \dot{x}(s)^{T} E^{T} Z E \dot{x}(s) d s \\
& \leq d e^{2 \alpha t} \dot{x}(t)^{T} E^{T} Z E \dot{x}(t) \\
& -e^{2 \alpha t} \int_{t-d}^{t} e^{-2 \alpha d} \dot{x}(s)^{T} E^{T} Z E \dot{x}(s) d s, \\
& \dot{V}_{4}(t)=\left(t_{k+1}-t\right) e^{2 \alpha t} \dot{x}(t)^{T} E^{T} U E \dot{x}(t) \\
& -\int_{t_{k}}^{t} e^{2 \alpha s} \dot{x}(s)^{T} E^{T} U E \dot{x}(s) d s \\
& \leq\left(t_{k+1}-t\right) e^{2 \alpha t} \dot{x}(t)^{T} E^{T} U E \dot{x}(t) \\
& -\int_{t_{k}}^{t} e^{2 \alpha t_{k}} \dot{x}(s)^{T} E^{T} U E \dot{x}(s) d s \\
& \leq\left(t_{k+1}-t\right) e^{2 \alpha t} \dot{x}(t)^{T} E^{T} U E \dot{x}(t) \\
& -e^{2 \alpha t} \int_{t_{k}}^{t} e^{-2 \alpha h} \dot{x}(s)^{T} E^{T} U E \dot{x}(s) d s, \\
& \dot{V}_{5}(t)=-\int_{t_{k}}^{t} e^{2 \alpha s}\left[\begin{array}{c}
x(s) \\
x\left(t_{k}\right)
\end{array}\right]^{T}\left[\begin{array}{cc}
R_{1} & R_{2} \\
* & R_{3}
\end{array}\right]\left[\begin{array}{c}
x(s) \\
x\left(t_{k}\right)
\end{array}\right] d s \\
& +\left(t_{k+1}-t\right) e^{2 \alpha t}\left[\begin{array}{c}
x(t) \\
x\left(t_{k}\right)
\end{array}\right]^{T}\left[\begin{array}{cc}
R_{1} & R_{2} \\
* & R_{3}
\end{array}\right]\left[\begin{array}{c}
x(t) \\
x\left(t_{k}\right)
\end{array}\right] \\
& \leq-\int_{t_{k}}^{t} e^{2 \alpha t_{k}}\left[\begin{array}{c}
x(s) \\
x\left(t_{k}\right)
\end{array}\right]^{T}\left[\begin{array}{cc}
R_{1} & R_{2} \\
* & R_{3}
\end{array}\right]\left[\begin{array}{c}
x(s) \\
x\left(t_{k}\right)
\end{array}\right] d s \\
& +\left(t_{k+1}-t\right) e^{2 \alpha t}\left[\begin{array}{c}
x(t) \\
x\left(t_{k}\right)
\end{array}\right]^{T}\left[\begin{array}{cc}
R_{1} & R_{2} \\
* & R_{3}
\end{array}\right]\left[\begin{array}{c}
x(t) \\
x\left(t_{k}\right)
\end{array}\right] \\
& \leq-e^{2 \alpha t} \int_{t_{k}}^{t} e^{-2 \alpha h}\left[\begin{array}{c}
x(s) \\
x\left(t_{k}\right)
\end{array}\right]^{T}\left[\begin{array}{cc}
R_{1} & R_{2} \\
* & R_{3}
\end{array}\right]\left[\begin{array}{c}
x(s) \\
x\left(t_{k}\right)
\end{array}\right] d s \\
& +\left(t_{k+1}-t\right) e^{2 \alpha t}\left[\begin{array}{c}
x(t) \\
x\left(t_{k}\right)
\end{array}\right]^{T}\left[\begin{array}{cc}
R_{1} & R_{2} \\
* & R_{3}
\end{array}\right]\left[\begin{array}{c}
x(t) \\
x\left(t_{k}\right)
\end{array}\right] \\
& =\left(t_{k+1}-t\right) e^{2 \alpha t}\left[\begin{array}{c}
x(t) \\
x\left(t_{k}\right)
\end{array}\right]^{T}\left[\begin{array}{cc}
R_{1} & R_{2} \\
* & R_{3}
\end{array}\right]\left[\begin{array}{c}
x(t) \\
x\left(t_{k}\right)
\end{array}\right] \\
& -e^{2 \alpha t} \int_{t_{k}}^{t} e^{-2 \alpha h} x(s)^{T} R_{1} x(s) d s \\
& -2 e^{2 \alpha t} x\left(t_{k}\right)^{T} e^{-2 \alpha h} R_{2}^{T} \int_{t_{k}}^{t} x(s) d s \\
& -e^{2 \alpha t}\left(t-t_{k}\right) x\left(t_{k}\right)^{T} e^{-2 \alpha h} R_{3} x\left(t_{k}\right), \\
& \dot{V}_{6}(t)=-e^{2 \alpha t}\left[\begin{array}{c}
x(t) \\
\int_{t_{k}}^{t} x(s) d s \\
x\left(t_{k}\right)
\end{array}\right]^{T} E^{T} \Gamma E\left[\begin{array}{c}
x(t) \\
\int_{t_{k}}^{t} x(s) d s \\
x\left(t_{k}\right)
\end{array}\right] \\
& +2 \alpha\left(t_{k+1}-t\right) e^{2 \alpha t}\left[\begin{array}{c}
x(t) \\
\int_{t_{k}}^{t} x(s) d s \\
x\left(t_{k}\right)
\end{array}\right]^{T}
\end{aligned}
$$

$$
\times E^{T} \Gamma E\left[\begin{array}{c}
x(t) \\
\int_{t_{k}}^{t} x(s) d s \\
x\left(t_{k}\right)
\end{array}\right]+2\left(t_{k+1}-t\right)
$$$$
\times e^{2 \alpha t}\left[\begin{array}{c}
x(t) \\
\int_{t_{k}}^{t} x(s) d s \\
x\left(t_{k}\right)
\end{array}\right]^{T} E^{T} \Gamma E\left[\begin{array}{c}
\dot{x}(t) \\
x(t) \\
0
\end{array}\right]
$$$$
\leq-e^{2 \alpha t}\left[\begin{array}{c}
x(t) \\
\int_{t_{k}}^{t} x(s) d s \\
x\left(t_{k}\right)
\end{array}\right]^{T} E^{T} \Gamma E\left[\begin{array}{c}
x(t) \\
\int_{t_{k}}^{t} x(s) d s \\
x\left(t_{k}\right)
\end{array}\right]
$$$$
+2 \alpha\left(t_{k+1}-t\right) e^{2 \alpha t}\left[\begin{array}{c}
x(t) \\
\int_{t_{k}}^{t} x(s) d s \\
x\left(t_{k}\right)
\end{array}\right]^{T}
$$$$
\times E^{T} \Gamma E\left[\begin{array}{c}
x(t) \\
\int_{t_{k}}^{t} x(s) d s \\
x\left(t_{k}\right)
\end{array}\right]
$$$$
+2\left(t_{k+1}-t\right) e^{2 \alpha t} x(t)^{T} E^{T} \frac{X_{1}+X_{2}}{2} E \dot{x}(t)
$$$$
+2\left(t_{k+1}-t\right) e^{2 \alpha t} x\left(t_{k}\right)^{T} E^{T}\left(-X_{1}^{T}+X_{2}^{T}\right) E \dot{x}(t)
$$$$
+2\left(t_{k+1}-t\right) e^{2 \alpha t} \int_{t_{k}}^{t} x(s)^{T} d s E^{T} X_{3}^{T} E \dot{x}(t)
$$$$
+2\left(t_{k+1}-t\right) e^{2 \alpha t} x(t)^{T} E^{T} X_{3} E x(t)
$$$$
+2\left(t_{k+1}-t\right) e^{2 \alpha t} x\left(t_{k}\right)^{T} E^{T} X_{4}^{T} E x(t)
$$$$
+2\left(t_{k+1}-t\right) e^{2 \alpha t} \int_{t_{k}}^{t} x(s)^{T} d s E^{T} \frac{X_{5}+X_{5}^{T}}{2} E x(t) .
$$

According to Jensen integral inequality [34], we have

$$
\begin{aligned}
-\int_{t-d}^{t} e^{-2 \alpha d} \dot{x}(s)^{T} E^{T} Z E \dot{x}(s) d s & \\
\leq & -\int_{t-d}^{t} \dot{x}(s)^{T} E^{T} d s \frac{e^{-2 \alpha d}}{d} Z \int_{t-d}^{t} E \dot{x}(s) d s \\
= & {\left[\begin{array}{c}
x(t) \\
x(t-d)
\end{array}\right]^{T}\left[\begin{array}{cc}
-\frac{e^{-2 \alpha d}}{d} E^{T} Z E & \frac{e^{-2 \alpha d}}{d} E^{T} Z E \\
* & -\frac{e^{-2 \alpha d}}{d} E^{T} Z E
\end{array}\right] } \\
& \times\left[\begin{array}{c}
x(t) \\
x(t-d)
\end{array}\right],
\end{aligned}
$$




$$
\begin{aligned}
-\int_{t_{k}}^{t} & e^{-2 \alpha h} x(s)^{T} R_{1} x(s) d s \\
& \leq-\int_{t_{k}}^{t} x(s)^{T} d s \frac{e^{-2 \alpha h}}{h} R_{1} \int_{t_{k}}^{t} x(s) d s .
\end{aligned}
$$

Applying (26) and (27) to (22) and (24), respectively, we can get

$$
\begin{aligned}
\dot{V}_{3}(t) \leq & d e^{2 \alpha t} \dot{x}(t)^{T} E^{T} Z E \dot{x}(t) \\
& +e^{2 \alpha t}\left[\begin{array}{c}
x(t) \\
x(t-d)
\end{array}\right]^{T} \\
& \times\left[\begin{array}{cc}
-\frac{e^{-2 \alpha d}}{d} E^{T} Z E & \frac{e^{-2 \alpha d}}{d} E^{T} Z E \\
* & -\frac{e^{-2 \alpha d}}{d} E^{T} Z E
\end{array}\right]\left[\begin{array}{c}
x(t) \\
x(t-d)
\end{array}\right],
\end{aligned}
$$

$$
\begin{aligned}
\dot{V}_{5}(t) \leq & \left(t_{k+1}-t\right) e^{2 \alpha t}\left[\begin{array}{c}
x(t) \\
x\left(t_{k}\right)
\end{array}\right]^{T}\left[\begin{array}{cc}
R_{1} & R_{2} \\
* & R_{3}
\end{array}\right]\left[\begin{array}{c}
x(t) \\
x\left(t_{k}\right)
\end{array}\right] \\
& -e^{2 \alpha t} \int_{t_{k}}^{t} x(s)^{T} d s \frac{e^{-2 \alpha h}}{h} R_{1} \int_{t_{k}}^{t} x(s) d s \\
& -2 e^{2 \alpha t} x\left(t_{k}\right)^{T} e^{-2 \alpha h} R_{2}^{T} \int_{t_{k}}^{t} x(s) d s \\
& -e^{2 \alpha t}\left(t-t_{k}\right) x\left(t_{k}\right)^{T} e^{-2 \alpha h} R_{3} x\left(t_{k}\right) .
\end{aligned}
$$

Furthermore, based on Schur complement, it can be found that for any appropriately dimensioned matrix $H$

$$
\left[\begin{array}{cc}
H^{T} e^{2 \alpha h} U^{-1} H & H^{T} \\
* & e^{-2 \alpha h} U
\end{array}\right] \geq 0
$$

which implies

$$
\int_{t_{k}}^{t}\left[\begin{array}{c}
\phi(t) \\
E \dot{x}(s)
\end{array}\right]^{T}\left[\begin{array}{cc}
H^{T} e^{2 \alpha h} U^{-1} H & H^{T} \\
* & e^{-2 \alpha h} U
\end{array}\right]\left[\begin{array}{c}
\phi(t) \\
E \dot{x}(s)
\end{array}\right] d s \geq 0,
$$

where

$$
\left.\phi(t)=\left[\begin{array}{lll}
x(t)^{T} & {[E \dot{x}(t)}
\end{array}\right]^{T} x\left(t_{k}\right)^{T} \int_{t_{k}}^{t} x(s)^{T} d s\right]^{T} .
$$

From (31), we can get

$$
\begin{aligned}
-\int_{t_{k}}^{t} e^{-2 \alpha h} \dot{x}(s)^{T} E^{T} U E \dot{x}(s) d s & \\
\leq & \left(t-t_{k}\right) \phi(t)^{T} H^{T} e^{2 \alpha h} U^{-1} H \phi(t) \\
& +2 \phi(t)^{T} H^{T} \operatorname{Ex}(t)-2 \phi(t)^{T} H^{T} \operatorname{Ex}\left(t_{k}\right) .
\end{aligned}
$$

Applying the above inequality to (23), we obtain

$$
\begin{aligned}
\dot{V}_{4}(t) \leq & e^{2 \alpha t}\left(t-t_{k}\right) \phi(t)^{T} E^{T} H^{T} e^{2 \alpha h} U^{-1} H E \phi(t) \\
& +2 e^{2 \alpha t} \phi(t)^{T} E^{T} H^{T} E x(t) \\
& -2 e^{2 \alpha t} \phi(t)^{T} E^{T} H^{T} E x\left(t_{k}\right) \\
& +e^{2 \alpha t}\left(t_{k+1}-t\right) \dot{x}(t)^{T} E^{T} U E \dot{x}(t) .
\end{aligned}
$$

On the other hand, according to (5), for any appropriately dimensioned matrix $F, F_{1}$, the following equation holds:

$$
\begin{aligned}
0= & 2 e^{2 \alpha t}\left[x(t)^{T} F^{T}+\dot{x}(t)^{T} E^{T} F_{1}^{T}\right] \\
& \times\left[-E \dot{x}(t)+A x(t)+A_{d} x(t-d)+K x\left(t_{k}\right)\right] .
\end{aligned}
$$

Then, adding the right-hand side of (35) to $\dot{V}(t)$, we obtain from (20), (21), (25), (28), (29), and (34) that for $t \in$ $\left[t_{k}, t_{k+1}\right)$

$$
\dot{V}(t) \leq e^{2 \alpha t} \chi(t)^{T}\left[\frac{t_{k+1}-t}{h_{k}} \Xi_{1}\left(h_{k}\right)+\frac{t-t_{k}}{h_{k}} \bar{\Xi}_{2}\left(h_{k}\right)\right] \chi(t),
$$

where $\bar{\Xi}_{2}\left(h_{k}\right)$ is given in (38), and

$$
\chi(t)=\left[\begin{array}{llll}
x(t)^{T} & {\left[\begin{array}{l}
E \dot{x}(t)
\end{array}\right]^{T} \quad x\left(t_{k}\right)^{T} \int_{t_{k}}^{t} x(s)^{T} d s \quad x(t-d)^{T}}
\end{array}\right]^{T}
$$

$$
\bar{\Xi}_{2}\left(h_{k}\right)=\left[\begin{array}{ccccc}
\Xi_{11} & \Xi_{12} & \Xi_{13} & \Xi_{14} & \Xi_{15} \\
* & \Xi_{22} & \Xi_{23} & 0 & F_{1} A_{d} \\
* & * & \Xi_{33}-e^{-2 \alpha h} \bar{h} R_{3} & \Xi_{34} & 0 \\
* & * & * & \Xi_{44} & 0 \\
* & * & * & * & \Xi_{55}
\end{array}\right]
$$

It is noted that

$$
\begin{aligned}
& \Xi_{1}\left(h_{k}\right)=\frac{h_{k}}{h} \Xi_{1}(h)+\frac{h-h_{k}}{h} \Xi_{1}(0), \\
& \bar{\Xi}_{2}\left(h_{k}\right)=\frac{h_{k}}{h} \bar{\Xi}_{2}(h)+\frac{h-h_{k}}{h} \bar{\Xi}_{2}(0) .
\end{aligned}
$$

From (12) and (13), we can find that

$$
\Xi_{1}\left(h_{k}\right)<0, \quad \Xi_{2}(0)<0 .
$$


Based on Schur complement, we have from (13)

$$
\bar{\Xi}_{2}(h)<0 \text {. }
$$

From (40), (41), we can get that

$$
\bar{\Xi}_{2}\left(h_{k}\right)<0 \text {. }
$$

Thus, we obtain from (36), (41), and (43) that

$$
\dot{V}(t)<0, \quad t \in\left[t_{k}, t_{k+1}\right) .
$$

Thus, it follows that, for $t \in\left[t_{k}, t_{k+1}\right)$,

$$
V(t) \leq V\left(t_{k}\right) \leq V\left(t_{k-1}\right) \leq \cdots \leq V(0) .
$$

Based on Lemma 3 and (45) and letting $\widehat{P}=E^{T} P E$, we can conclude that for $t_{k} \leq t<t_{k+1}$

$\|E x(t)\|^{2}$

$$
\begin{aligned}
\leq & \theta_{1}\left\|x\left(t_{k}\right)\right\|^{2}+\theta_{2} \int_{t_{k}-d}^{t_{k}}\|x(\alpha)\|^{2} d \alpha \\
= & \frac{\theta_{1}}{\lambda_{\min }(\widehat{P}) e^{2 \alpha t_{k}}} e^{2 \alpha t_{k}} \lambda_{\min }(\widehat{P})\left\|x\left(t_{k}\right)\right\|^{2} \\
& +\frac{\theta_{2}}{\lambda_{\min }(Q) e^{2 \alpha t_{k}}} e^{2 \alpha t_{k}} \lambda_{\min }(Q) \int_{t_{k}-d}^{t_{k}}\|x(\alpha)\|^{2} d \alpha \\
\leq & \frac{\theta_{1}}{\lambda_{\min }(\widehat{P}) e^{2 \alpha t_{k}} e^{2 \alpha t_{k}} x\left(t_{k}\right)^{T} \widehat{P} x\left(t_{k}\right)} \\
& +\frac{\theta_{2} e^{2 \alpha d}}{\lambda_{\min }(Q) e^{2 \alpha t_{k}}} \int_{t_{k}-d}^{t_{k}} e^{2 \alpha s} x(s)^{T} Q x(s) d s \\
\leq & \frac{\max \left\{\theta_{1} / \lambda_{\min }(\widehat{P}), \theta_{2} e^{2 \alpha d} / \lambda_{\min }(Q)\right\}}{e^{2 \alpha t_{k}}}\left(V_{1}\left(t_{k}\right)+V_{2}\left(t_{k}\right)\right) \\
\leq & \frac{\max \left\{\theta_{1} / \lambda_{\min }(\widehat{P}), \theta_{2} e^{2 \alpha d} / \lambda_{\min }(Q)\right\}}{e^{2 \alpha t_{k}}} V\left(t_{k}\right) \\
\leq & \max \left\{\frac{\theta_{1}}{\lambda_{\min }(\widehat{P})}, \frac{\theta_{2} e^{2 \alpha d}}{\lambda_{\min }(Q)}\right\} e^{-2 \alpha t} e^{2 \alpha\left(t-t_{k}\right)} V(0) \\
\lambda_{\min }(\widehat{P}) & \left.\frac{\theta_{2} e^{2 \alpha d}}{\lambda_{\min }(Q)}\right\} e^{-2 \alpha t} V(0)
\end{aligned}
$$

It can be calculated that

$$
\begin{aligned}
V(0)= & x(0)^{T} E^{T} P E x(0)+\int_{-d}^{0} e^{2 \alpha s} x(s)^{T} Q x(s) d s \\
& +\int_{-d}^{0} \int_{\theta}^{0} e^{2 \alpha s} \dot{x}(s)^{T} E^{T} Z E \dot{x}(s) d s d \theta \\
\leq & x(0)^{T} E^{T} P E x(0) \\
& +\int_{-d}^{0} x(s)^{T} Q x(s) d s+d \int_{-d}^{0} \dot{x}(s)^{T} E^{T} Z E \dot{x}(s) d s \\
\leq & \lambda_{\max }(\widehat{P})\|x(0)\|^{2}+d \lambda_{\max }(Q) \sup _{-d \leq \theta \leq 0}\|x(\theta)\|^{2}
\end{aligned}
$$

$$
\begin{gathered}
+d^{2} \lambda_{\max }(Z) \sup _{-d \leq \theta \leq 0}\|E \dot{x}(\theta)\|^{2} \\
\leq \theta_{3}\left(\sup _{-d \leq \theta \leq 0}\{\|x(\theta)\|,\|E \dot{x}(\theta)\|\}\right)^{2},
\end{gathered}
$$

where

$$
\theta_{3}=\lambda_{\max }(\widehat{P})+d \lambda_{\max }(Q)+d^{2} \lambda_{\max }(Z) .
$$

Based on (46) and (47), we can conclude that

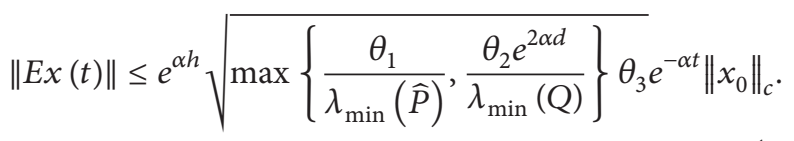

Thus, according to Definition 2, the sampled-data control for singular system (5) is exponentially admissible. This completes the proof.

Remark 5. It is noted that based on the time-dependent Lyapunov functional method, three $\left(t_{k}, t_{k+1}\right)$-dependent terms $V_{4}(t), V_{5}(t)$, and $V_{6}(t)$ are introduced in the Lyapunov functional, which make good use of the available information about the actual sampling pattern. As a consequence, the proposed result has less conservatism.

Remark 6. It should be pointed out that if $R_{1}=R_{3}=\varepsilon I$ ( $\varepsilon$ is a sufficiently small positive scalar) and $R_{2}=X_{3}=X_{4}=X_{5}=$ 0 , then $V_{4}(t)+V_{5}(t)+V_{6}(t)$ reduces to

$$
V_{4}(t)+\left(t_{k+1}-t\right) e^{2 \alpha t}\left[\begin{array}{c}
x(t) \\
x\left(t_{k}\right)
\end{array}\right]^{T} E^{T} \Psi E\left[\begin{array}{c}
x(t) \\
x\left(t_{k}\right)
\end{array}\right]
$$

where

$$
\Psi=\left[\begin{array}{cc}
\frac{X_{1}+X_{1}^{T}}{2} & -X_{1}+X_{2} \\
* & -X_{2}-X_{2}^{T}+\frac{X_{1}+X_{1}^{T}}{2}
\end{array}\right],
$$

which was first proposed for linear sampled-data systems in [16]. On the other hand, in [16] the Lyapunov functional should be positive definite at the whole sampling intervals. While the Lyapunov functional (17) is positive definite only at sampling times but not necessarily positive definite inside the sampling intervals. Thus, the Lyapunov functional used in this paper is more general and desirable than the one adopted in [16]. lary.

Based on Theorem 4, we can obtain the following corol-

Corollary 7. If (12) and (13) are feasible for $\alpha=0$, then the system (5) is exponentially stable with a small enough decay rate.

Now, we will design the sampled-data controller (4) such that system (5) is exponentially stable. Based on Theorem 4, the sampled-data controller design method for system (5) is provided in the following theorem. 
Theorem 8. Given scalars $\alpha>0$ and $\lambda>0$, the sampleddata control for singular system (5) is exponentially stabile if there exist symmetric positive-definite matrices $P, F, Q, Z, U$, $\left[\begin{array}{ll}R_{1} & R_{2} \\ * & R_{3}\end{array}\right]>0, X_{j}, j=1,2,3,4,5, L, H=\left[\begin{array}{llll}H_{1} & H_{2} & H_{3} & H_{4}\end{array}\right]$, such that

$$
\begin{aligned}
& \widetilde{\Xi}_{1}(\bar{h})=\left[\begin{array}{ccccc}
\Xi_{11}+\Theta_{11}(\bar{h}) & \widetilde{\Xi}_{12}+\Theta_{12}(\bar{h}) & \widetilde{\Xi}_{13}+\Theta_{13}(\bar{h}) & \Xi_{14}+\Theta_{14}(\bar{h}) & \Xi_{15} \\
* & \widetilde{\Xi}_{22}+\bar{h} U & \widetilde{\Xi}_{23}+\Theta_{23}(\bar{h}) & \bar{h} E^{T} X_{3} E & \lambda F A_{d} \\
* & * & \Xi_{33}+\Theta_{33}(\bar{h}) & \Xi_{34}+2 \alpha \bar{h} X_{4} & 0 \\
* & * & * & \Xi_{44}+\Theta_{44}(\bar{h}) & 0 \\
* & * & * & * & \Xi_{55}
\end{array}\right]<0 \\
& \widetilde{\Xi}_{2}(\bar{h})=\left[\begin{array}{cccccc}
\Xi_{11} & \widetilde{\Xi}_{12} & \widetilde{\Xi}_{13} & \Xi_{14} & \Xi_{15} & \sqrt{\bar{h}} E^{T} H_{1}^{T} E \\
* & \widetilde{\Xi}_{22} & \widetilde{\Xi}_{23} & 0 & \lambda F A_{d} & \sqrt{\bar{h}} E^{T} H_{2}^{T} E \\
* & * & \Xi_{33}-e^{-2 \alpha h} \bar{h} R_{3} & \Xi_{34} & 0 & \sqrt{\bar{h}} E^{T} H_{3}^{T} E \\
* & * & * & \Xi_{44} & 0 & \sqrt{\bar{h}} E^{T} H_{4}^{T} E \\
* & * & * & * & \Xi_{55} & 0 \\
* & * & * & * & * & -e^{-2 \alpha h} E^{T} U E
\end{array}\right]<0,
\end{aligned}
$$

where $\Xi_{11}, \Xi_{14}, \Xi_{15}, \Xi_{33}, \Xi_{34}, \Xi_{44}, \Xi_{55}, \Theta_{11}(\bar{h}), \Theta_{12}(\bar{h}), \Theta_{13}(\bar{h})$, $\Theta_{14}(\bar{h}), \Theta_{23}(\bar{h}), \Theta_{33}(\bar{h}), \Theta_{44}(\bar{h})$ are as those in Theorem 4 , and

$$
\begin{aligned}
& \widetilde{\Xi}_{12}=E^{T} P+E^{T} H_{2} E-F^{T}+A^{T} \lambda F^{T}, \\
& \widetilde{\Xi}_{13}=E^{T}\left(X_{1}-X_{2}\right) E+E^{T}\left(H_{3}-H_{1}^{T}\right) E+L, \\
& \widetilde{\Xi}_{22}=d Z-\lambda F-\lambda F^{T}, \\
& \widetilde{\Xi}_{23}=-E^{T} H_{2}^{T} E-\lambda L .
\end{aligned}
$$

Then singular system (5) is exponentially stable. Furthermore, the sampled-data controller gain matrix in (5) is given by

$$
K=F^{-T} L
$$

Proof. Letting $F_{1}=\lambda F$, and $F^{T} K=L$, we can get from (12)(13) that (52)-(53) hold. This completes the proof.

Remark 9. It should be mentioned that the problem of sampled-data exponential stability of singular systems with time constant delays and uncertain sampling is solved in Theorem 8, and sufficient conditions of the existence of the desired sampled-data controllers are also given, which are formulated by LMIs and can readily be solved by standard numerical software. lary.
TABLE 1: Maximum values of the upper bound $h$ for different $\alpha$.

\begin{tabular}{cccccc}
\hline$\alpha$ & 0.1 & 0.2 & 0.3 & 0.4 & 0.5 \\
\hline$h$ & 0.3450 & 0.3150 & 0.2760 & 0.2390 & 0.2080 \\
\hline
\end{tabular}

Corollary 10. If (12), (13) and (52), (53) are feasible for $\alpha=$ 0 , then system (5) is exponentially stable with a small enough decay rate, and the desired state feedback controller gains are given in (55).

\section{Numerical Examples}

In this section, two illustrative examples will be provided to demonstrate the validity and reduced conservatism of the proposed approaches.

Example 1. Consider the singular system with sampled-data control in (5). The system parameters are described as follows:

$$
\begin{gathered}
A=\left[\begin{array}{ccc}
-2.5714 & 9 & 0 \\
1 & -1 & 1 \\
0 & 13.95 & 0
\end{array}\right], \\
A_{d}=\left[\begin{array}{ccc}
-0.1 & 0 & 0 \\
-0.1 & 0 & 0 \\
0.2 & 0 & -0.1
\end{array}\right], \quad E=\left[\begin{array}{lll}
1 & 0 & 0 \\
0 & 1 & 0 \\
0 & 0 & 0
\end{array}\right] .
\end{gathered}
$$

In this example, we choose $d=1, \lambda=0.2$.

Applying Theorem 4, as shown in Table 1, we can obtain the different maximum values of the upper bound $h$ for 


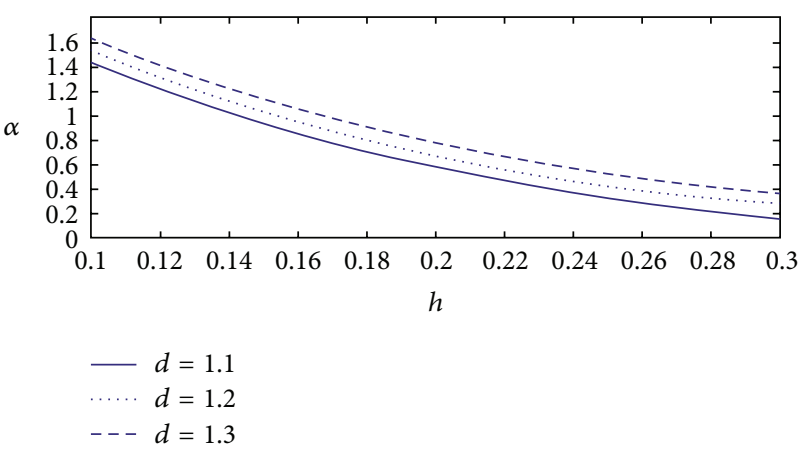

Figure 1: The upper bound $h$ for different $\alpha$.

different $\alpha$. From Table 1, we can find the influence of the choice of $\alpha$ on the value of the upper bound $h$. To be specific, a larger value of $\alpha$ corresponds to a smaller value of the upper bound $h$.

Next, we will design the sampled-data controller (4) such that system (5) is exponentially stable. Choosing $\alpha=0.4$ and $h=0.2390$, and using the MATLAB LMI Toolbox to solve the LMIs (12) and (13), we can get the following gain matrix in

$$
K=\left[\begin{array}{ccc}
-0.1937 & -0.2703 & -0.1003 \\
-3.4469 & -4.8445 & -0.0004 \\
1.6194 & 2.2752 & 0.0011
\end{array}\right]
$$

That is, there exists a sampled-data controller such that system (5) is exponentially stable for any sampling period $h_{k} \leq 0.2390$.

For the case of constant sampling period, based on Theorem 1 of [35], the maximum sampling period $h$ is 0.0158 . While based on Corollary 10 with $\alpha=0$, the largest sampling period $h$ ensuring the stability of system (9) is 0.0389 , which is $146.2 \%$ larger than that of [35]. Thus, our proposed approach is able to achieve less conservative results and essentially improves the existing one.

Example 2. Consider the singular system with sampled-data control in (5) with the following parameters:

$$
\begin{gathered}
A=\left[\begin{array}{ll}
-0.6 & 0.54 \\
-0.6 & 0.12
\end{array}\right], \\
A_{d}=\left[\begin{array}{cc}
-0.5 & 0.4 \\
0.5 & -0.1
\end{array}\right], \quad E=\left[\begin{array}{ll}
1 & 0 \\
0 & 0
\end{array}\right] .
\end{gathered}
$$

In this example, we choose $\lambda=0.3$.

For different time-delay $d$, the influence of the choice of the upper bound $h$ on the value of $\alpha$ can be seen in Figure 1. From Figure 1, it is clear that when time-delay $d$ is fixed, for a larger upper bound $h$, the value of $\alpha$ is usually smaller, and when $h$ is fixed, for a larger $d$, the value of $\alpha$ is usually larger.

Using Theorem 8 with $\alpha=0.06$ given in this paper, the maximum value of the upper bound $h$ that system (5) is exponentially stable is 0.3029 , and the corresponding gain matrix is

$$
K=\left[\begin{array}{cc}
-3.9755 & 0.0646 \\
2.1741 & -0.0142
\end{array}\right]
$$

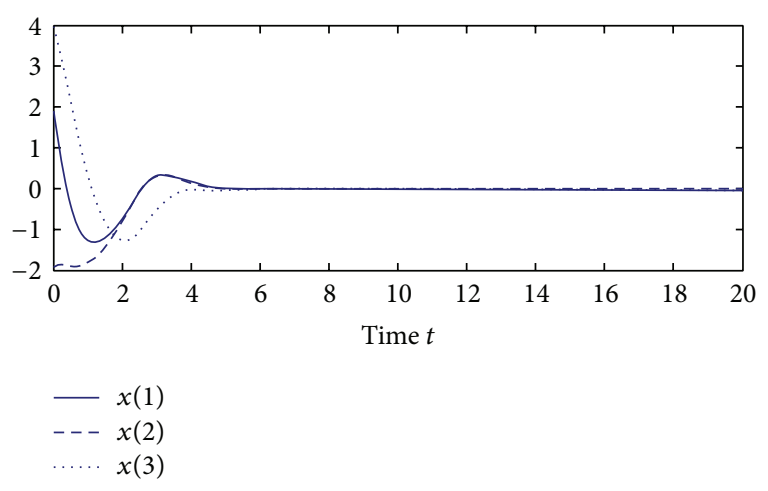

FIGURE 2: State response of system (5).

Under the above gain matrix, the response curves of system (5) are exhibited in Figure 2, which shows that the states are tending to zero; that is, singular system (5) can be stabilized by the proposed sampled-data controller.

\section{Conclusion}

In this paper, a sampled-data control approach was proposed for the singular systems with time delays. A time-dependent Lyapunov functional was introduced for the systems, which was positive definite at sampling times but not necessarily positive definite inside the sampling intervals. By the usage of the Lyapunov approach, sufficient condition was proposed to ensure the exponential stability of the singular systems, which can significantly reduce the conservatism. The available information about the actual sampling pattern was fully used. Based on the stability criterion, the desired sampled-data controller has also been designed. Finally, two illustrative examples have been presented to show the effectiveness and potential of the proposed new design techniques.

\section{Conflict of Interests}

The authors declare that there is no conflict of interests regarding the publication of this paper.

\section{Acknowledgments}

This work was supported by the National Natural Science Foundation of China (51109090), The key project of Fujian Provincial Department of Science \& Technology (2012H0030), the university's innovative project of Xiamen Science \& Technology Bureau (3502Z20123019), and the project of New Century Excellent Talents of Colleges and Universities of Fujian Province (JA12181).

\section{References}

[1] P. Shi, "Filtering on sampled-data systems with parametric uncertainty," IEEE Transactions on Automatic Control, vol. 43, no. 7, pp. 1022-1027, 1998.

[2] S. K. Nguang and P. Shi, "Fuzzy $H_{\infty}$ output feedback control of nonlinear systems under sampled measurements," Automatica, vol. 39, no. 12, pp. 2169-2174, 2003. 
[3] E. Fridman, A. Seuret, and J.-P. Richard, "Robust sampleddata stabilization of linear systems: an input delay approach," Automatica, vol. 40, no. 8, pp. 1441-1446, 2004.

[4] L. Hu, P. Shi, and P. M. Frank, "Robust sampled-data control for Markovian jump linear systems," Automatica, vol. 42, no. 11, pp. 2025-2030, 2006.

[5] H. Gao, J. Wu, and P. Shi, "Robust sampled-data $H_{\infty}$ control with stochastic sampling," Automatica, vol. 45, no. 7, pp. 17291736, 2009.

[6] Q. Liu, R. Wang, and D. Wu, "Stability analysis for sampleddata systems based on multiple lyapunov functional method," International Journal of Innovative Computing Information and Control, vol. 8, no. 9, pp. 6345-6355, 2012.

[7] I. Mizumoto, Y. Fujimoto, N. Watanabe, and Z. Iwai, "Fast rate adaptive output feedback control of multi-rate sampled systems with an adaptive output estimator," International Journal of Innovative Computing, Information and Control, vol. 7, no. 7, pp. 4377-4394, 2011.

[8] A. Seuret, "A novel stability analysis of linear systems under asynchronous samplings," Automatica, vol. 48, no. 1, pp. 177182, 2012.

[9] W. Zhang, M. S. Branicky, and S. M. Phillips, "Stability of networked control systems," IEEE Control Systems Magazine, vol. 21, no. 1, pp. 84-97, 2001.

[10] L. Hu, J. Lam, Y. Cao, and H. Shao, "A linear matrix inequality (LMI) approach to robust $\mathrm{H} 2$ sampled-data control for linear uncertain systems," IEEE Transactions on Systems, Man, and Cybernetics B, vol. 33, no. 1, pp. 149-155, 2003.

[11] E. Fridman, A. Seuret, and J.-P. Richard, "Robust sampleddata stabilization of linear systems: an input delay approach," Automatica, vol. 40, no. 8, pp. 1441-1446, 2004.

[12] Z. Wu, P. Shi, H. Su, and J. Chu, "Stochastic synchronization of markovian jump neural networks with time-varying delay using sampled data," IEEE Transactions on Cybernetics, vol. 43, no. 6, pp. 1796-1806, 2013.

[13] B. Shen, Z. Wang, and X. Liu, "A stochastic sampled-data approach to distributed $H_{\infty}$ filtering in sensor networks," IEEE Transactions on Circuits and Systems, vol. 58, no. 9, pp. 22372246, 2011.

[14] B. Shen, Z. Wang, and X. Liu, "Sampled-data synchronization control of dynamical networks with stochastic sampling," IEEE Transactions on Automatic Control, vol. 57, no. 10, pp. 26442650, 2012.

[15] J. Li, J. Wu, S. Wang, and J. Cui, "Stability and stabilization for sampled-data systems with probabilistic sampling," International Journal of Innovative Computing, Information and Control, vol. 6, no. 7, pp. 3299-3311, 2010.

[16] E. Fridman, "A refined input delay approach to sampled-data control," Automatica, vol. 46, no. 2, pp. 421-427, 2010.

[17] E. Fridman and A. Blighovsky, "Robust sampled-data control of a class of semilinear parabolic systems," Automatica, vol. 48, no. 5, pp. 826-836, 2012.

[18] Z. Wu, P. Shi, H. Su, and J. Chu, "Exponential synchronization of neural networks with discrete and distributed delays under time-varying sampling," IEEE Transactions on Neural Networks and Learning Systems, vol. 23, no. 9, pp. 1368-1376, 2012.

[19] Z. Wu, P. Shi, H. Su, and J. Chu, "Network-based robust passive control for fuzzy systems with randomly occurring uncertainties," IEEE Transactions on Fuzzy Systems, vol. 21, no. 5, pp. 966-971, 2012.
[20] K. Liu, E. Fridman, and L. Hetel, "Stability and $L_{2}$-gain analysis of networked control systems under Round-Robin scheduling: a time-delay approach," Systems \& Control Letters, vol. 61, no. 5, pp. 666-675, 2012.

[21] K. Liu and E. Fridman, "Wirtinger's inequality and Lyapunovbased sampled-data stabilization," Automatica, vol. 48, no. 1, pp. 102-108, 2012.

[22] A. Seuret, "A novel stability analysis of linear systems under asynchronous samplings," Automatica, vol. 48, no. 1, pp. 177182,2012

[23] A. Seuret, "Stability analysis for sampled-data systems with a time-varying period," in Proceedings of the 48th IEEE Conference on Decision and Control held jointly with 2009 28th Chinese Control Conference (CDC/CCC '09), pp. 8130-8135, December 2009.

[24] W. Jiang and A. Seuret, "Improved stability analysis of networked control systems under asynchronous sampling and input delay," in Proceedings of the 2nd IFAC Workshop on Distributed Estimation and Control in Networked Systems (NecSys '10), pp. 79-84, September 2010.

[25] Z.-G. Wu, J. H. Park, H. Su, and J. Chu, "Stochastic stability analysis for discrete-time singular Markov jump systems with timevarying delay and piecewise-constant transition probabilities," Journal of the Franklin Institute, vol. 349, no. 9, pp. 2889-2902, 2012.

[26] P. Liu, "Further results on the exponential stability criteria for time delay singular systems with delay-dependence," International Journal of Innovative Computing, Information and Control, vol. 8, no. 6, pp. 4015-4024, 2012.

[27] P. Liu, "Further results on the exponential stability criteria for time delay singular systems with delay-dependence," International Journal of Innovative Computing, Information and Control, vol. 8, no. 6, pp. 4015-4024, 2012.

[28] H. Wanga, B. Zhoua, R. Lub, and A. Xueb, "New stability and stabilization criteria for a class of fuzzy singular systems with time-varying delay," Journal of the Franklin Institute, 2013.

[29] Y. Wang, Q. Wang, P. Zhou, and D. Duan, "Robust guaranteed cost control for singular Markovian jump systems with timevarying delay," ISA Transactions, vol. 51, no. 5, pp. 559-565, 2012.

[30] Y. Wang, P. Shi, Q. Wang, and D. Duan, "Exponential $H_{\infty}$ filtering for singular Markovian jump systems with mixed mode-dependent time-varying delay," IEEE Transactions on Circuits and Systems, vol. 60, no. 9, pp. 2440-2452, 2013.

[31] R. Loxton, K. L. Teo, and V. Rehbock, "An optimization approach to state-delay identification," IEEE Transactions on Automatic Control, vol. 55, no. 9, pp. 2113-2119, 2010.

[32] Q. Chai, R. Loxton, K. L. Teo, and C. Yang, "A unified parameter identification method for nonlinear time-delay systems," Journal of Industrial and Management Optimization, vol. 9, no. 2, pp. 471-486, 2013.

[33] Z. Wu, H. Su, and J. Chu, "Delay-dependent $H_{\infty}$ control for singular Markovian jump systems with time delay," Optimal Control Applications \& Methods, vol. 30, no. 5, pp. 443-461, 2009.

[34] K. Gu, V. L. Kharitonov, and J. Chen, Stability of Time-Delay Systems, Birkhäuser, Boston, Mass, USA, 2003.

[35] H. K. Lam and F. H. F. Leung, "Stabilization of chaotic systems using linear sampled-data controller," International Journal of Bifurcation and Chaos in Applied Sciences and Engineering, vol. 17, no. 6, pp. 2021-2031, 2007. 


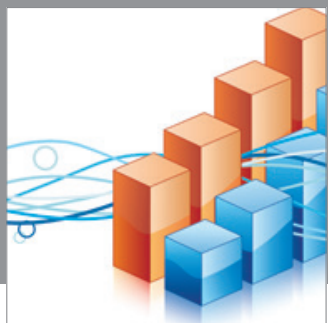

Advances in

Operations Research

mansans

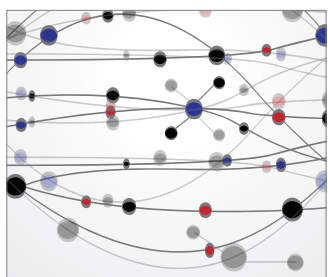

The Scientific World Journal
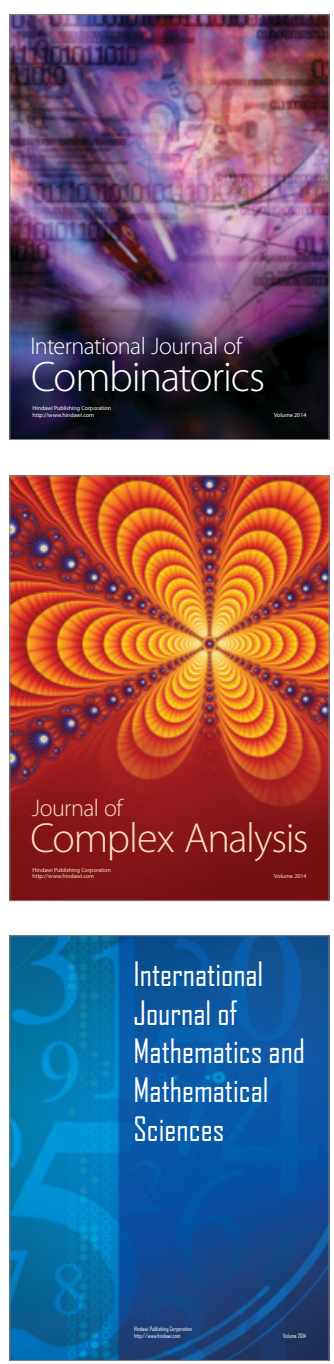
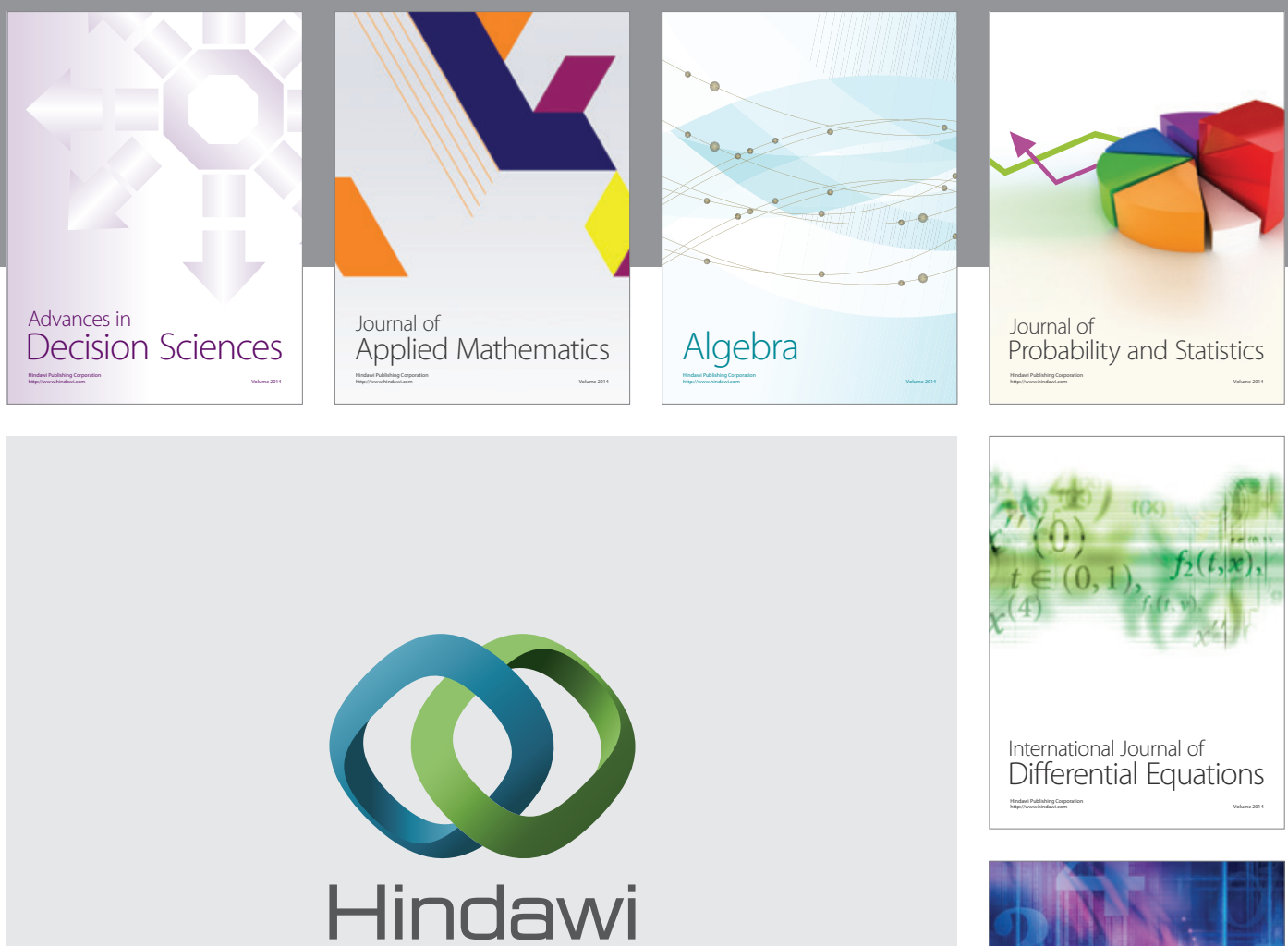

Submit your manuscripts at http://www.hindawi.com
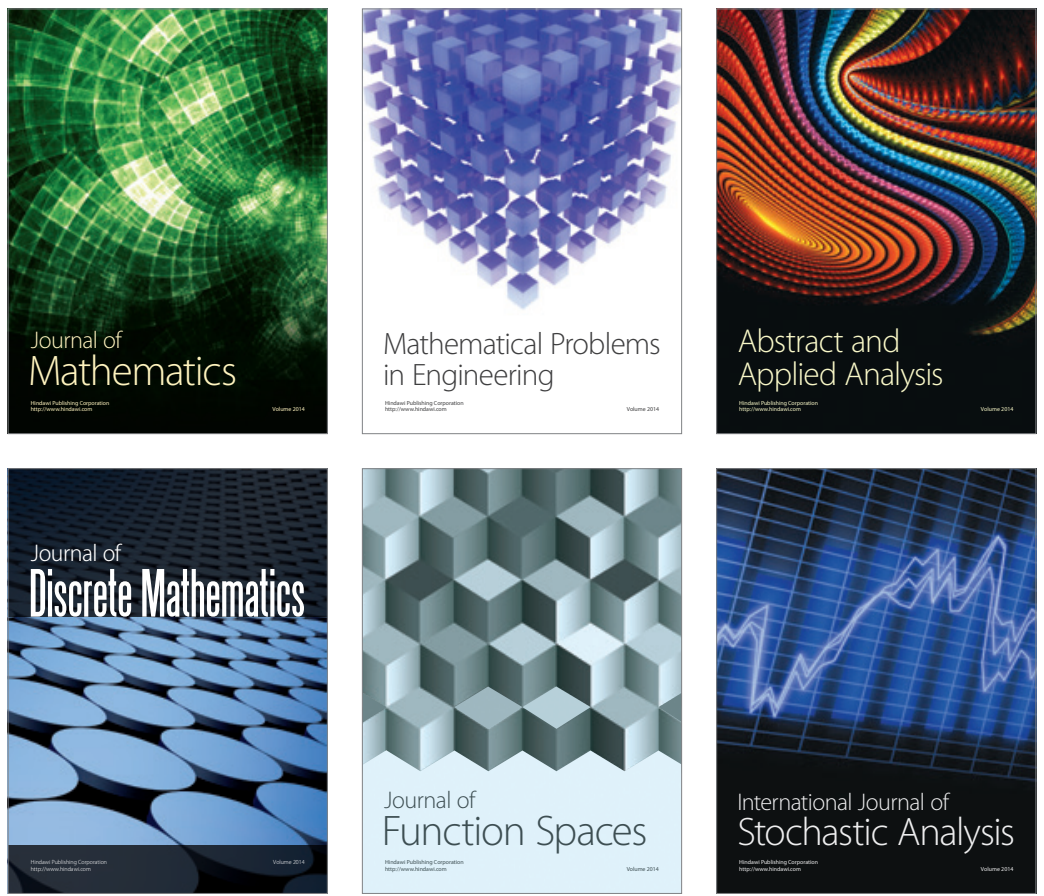

Journal of

Function Spaces

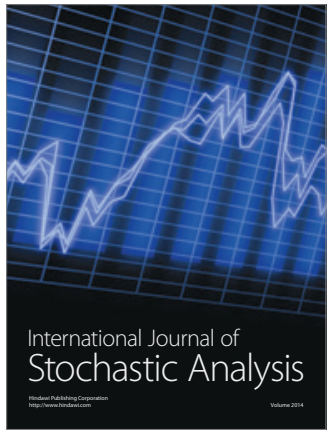

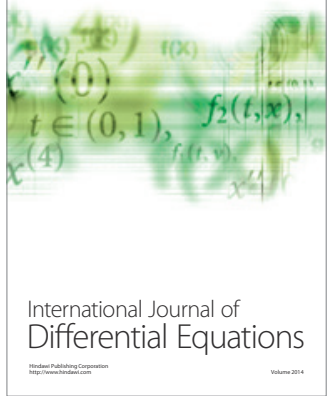
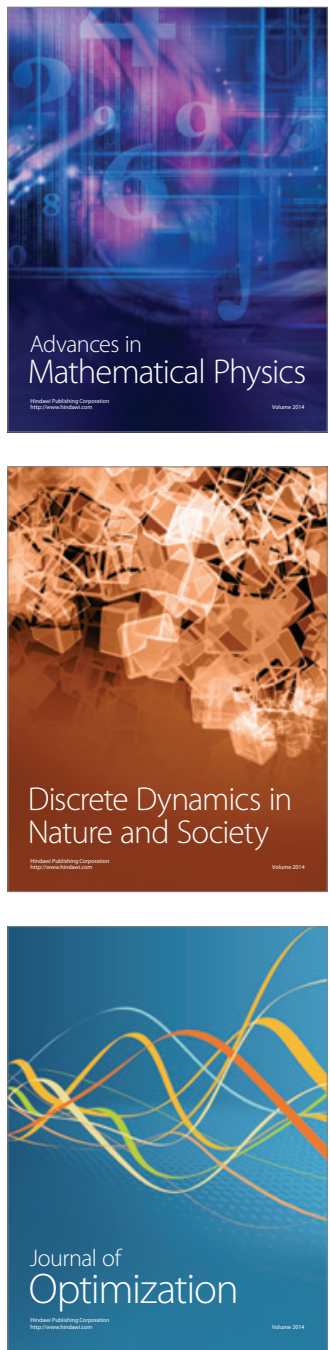